\title{
Guía para el seguimiento del desarrollo infantil en la práctica pediátrica
}

\author{
Guide for monitoring children's development in pediatric practice
}

Comité de Crecimiento y Desarrollo*

\begin{abstract}
RESUMEN
El pediatra es el médico de cabecera de los niños y es el primero en ser consultado cuando surgen preocupaciones en el desarrollo. La identificación temprana, el diagnóstico y el seguimiento de estos pacientes constituyen un desafío cuando se atiende a un niño pequeño. Esta guía ofrece recomendaciones para la vigilancia del desarrollo, la pesquisa, la evaluación y el seguimiento de pacientes con retrasos y discapacidades del desarrollo.

Palabras clave: crecimiento y desarrollo, discapacidades del desarrollo, encuestas de atención de la salud, humanos, lactante, preescolar.
\end{abstract}

Texto completo como Suplemento de Archivos Argentinos de Pediatría, en formato electrónico, en su sitio web (véase sección "Suplementos).

\begin{abstract}
The pediatrician is the best-informed professional to whom many families look to be the expert, not only on childhood illnesses but also on development. Early identification, diagnosis and monitoring of these patients are a real challenge for physicians who serve children. This guide provides recommendations for development monitoring, evaluation, diagnosis and follow up of patients with developmental delays and disabilities.

Key words: growth and development, developmental disabilities/ diagnosis, developmental disabilities/epidemiology, health care surveys, humans, infant, child, preschool.
\end{abstract}

http: / / dx.doi.org/10.5546/ aap.2017.s53

Cómo citar: Comité de Crecimiento y Desarrollo. Guía para el seguimiento del desarrollo infantil en la práctica pediátrica. Arch Argent Pediatr 2017;115 Supl 3:s53-s62.

\section{Restricción del crecimiento intrauterino: perspectiva endocrinológica Actualización de octubre de 2016 de la publicación de Archivos Argentinos de Pediatría 2007;105(1):71-73 \\ Intrauterine growth restriction: endocrinological perspective Update of the 2007 version (Arch Argent Pediatr 2007;105(1):71-73)}

\section{Comité Nacional de Endocrinología}

\section{RESUMEN}

La mayoría de los niños nacidos con bajo peso o restricción del crecimiento intrauterino desarrollan un crecimiento compensador que les permite alcanzar una talla final acorde a su talla objetivo genética. Sin embargo, en alrededor de un $15 \%$ de los niños, este crecimiento es insuficiente.

Tanto en los niños que presentan un adecuado crecimiento compensador y, por lo tanto, talla normal como en los niños que persisten bajos en la infancia, es necesario tener en cuenta que, si la pubertad se inicia en forma temprana, el pronóstico de talla final podría verse comprometido. Una intervención oportuna y adecuada podría mejorar la talla final.

También es importante el control del peso corporal, ya que su excesiva ganancia en la infancia se asocia a complicaciones metabólicas en la adolescencia y la vida adulta.

Palabras clave: retardo del crecimiento intrauterino, crecimiento, pubertad, enfermedades metabólicas.

Texto completo como Suplemento de Archivos Argentinos de Pediatría, en formato electrónico, en su sitio web (véase sección "Suplementos).

\begin{abstract}
Most children born with low weight or intrauterine growth restriction develop catch-up growth that allows them to reach a final height according to their genetic target height. However, in about $15 \%$ of children this growth is insufficient.

In both, children who have adequate compensatory growth and therefore normal height and children who remain low in childhood, it is necessary to take into account that if puberty starts early, the final height could be compromised. Timely and appropriate intervention could improve it.

Control of body weight is also important, as an excessive gain in childhood is associated with metabolic complications in adolescence and adult life.

Key words: intrauterine growth restriction, growth, puberty, metabolic alterations.
\end{abstract}

http:/ / dx.doi.org/10.5546/ aap.2017.s63.

Cómo citar: Comité Nacional de Endocrinología. Restricción del crecimiento intrauterino: perspectiva endocrinológica. Arch Argent Pediatr 2017;115 Supl 3: s63-s67. 